\title{
VariableR Reclustering in Multiple
}

Top Quark Events

\section{Jeremy Hyde}

Office of Science, Science Undergraduate Laboratory Internship (SULI) Program

This work was supported in part by the U.S. Department of Energy, Office of Science, Office of Workforce Development for Teachers and Scientists (WDTS) under the Science Undergraduate Laboratory Internship (SULI) program, under Contract No. DE-AC02-76SF00515. 


\section{Outline}

- Introduction

- LHC and ATLAS Detector

- Jets

- Boosted Objects

- Reclustering

- VariableR vs Fixed

- Top Quark Reconstruction

- W Boson Reconstruction

- Comparison 


\section{Absract}

VariableR jet reclustering is an innovative technique that allows for the reconstruction of boosted object over a wide range of kinematic regimes. Such capability enables the efficient identification of events with multiple boosted top quarks which is a typical signature for new physics processes such as the production of the supersymmetric partner of the gluon. In order to evaluate the performance of the algorithm, the VariableR reclustered jets are compared with fixed radius reclustered jets. The flexibility of the algorithm is tested by reconstructing both boosted top quarks and boosted W bosons. The VariableR reclustering method is found to be more efficient than the fixed radius algorithm at identifying top quarks and $\mathrm{W}$ bosons in events with four top quarks, therefore enhancing the sensitivity for gluino searches. 


\section{Introduction}

- Looking at multiple top quark events in order to study new physics such as super symmetry

- In particular the top quarks decay into a $\mathrm{W}$ boson and bottom quark

- Gluinos can be very massive so their decay produces can be very energetic

- Key for signal discrimination is efficient reconstruction of top (or W) multiplicity.
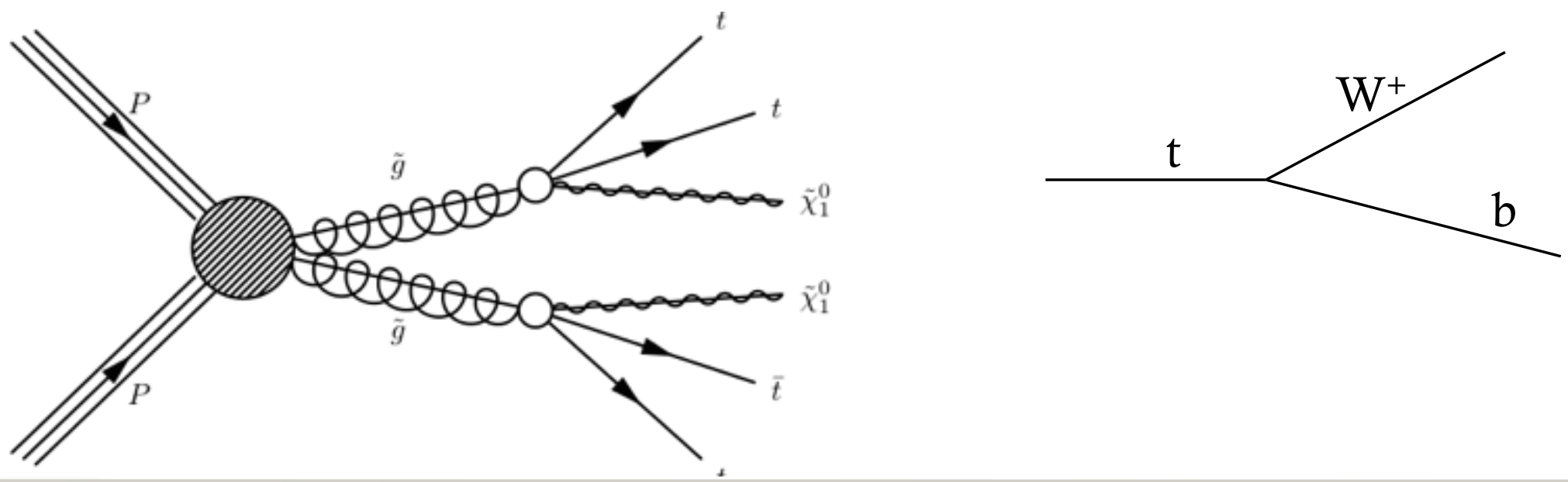


\section{LHC and ATLAS Detector}

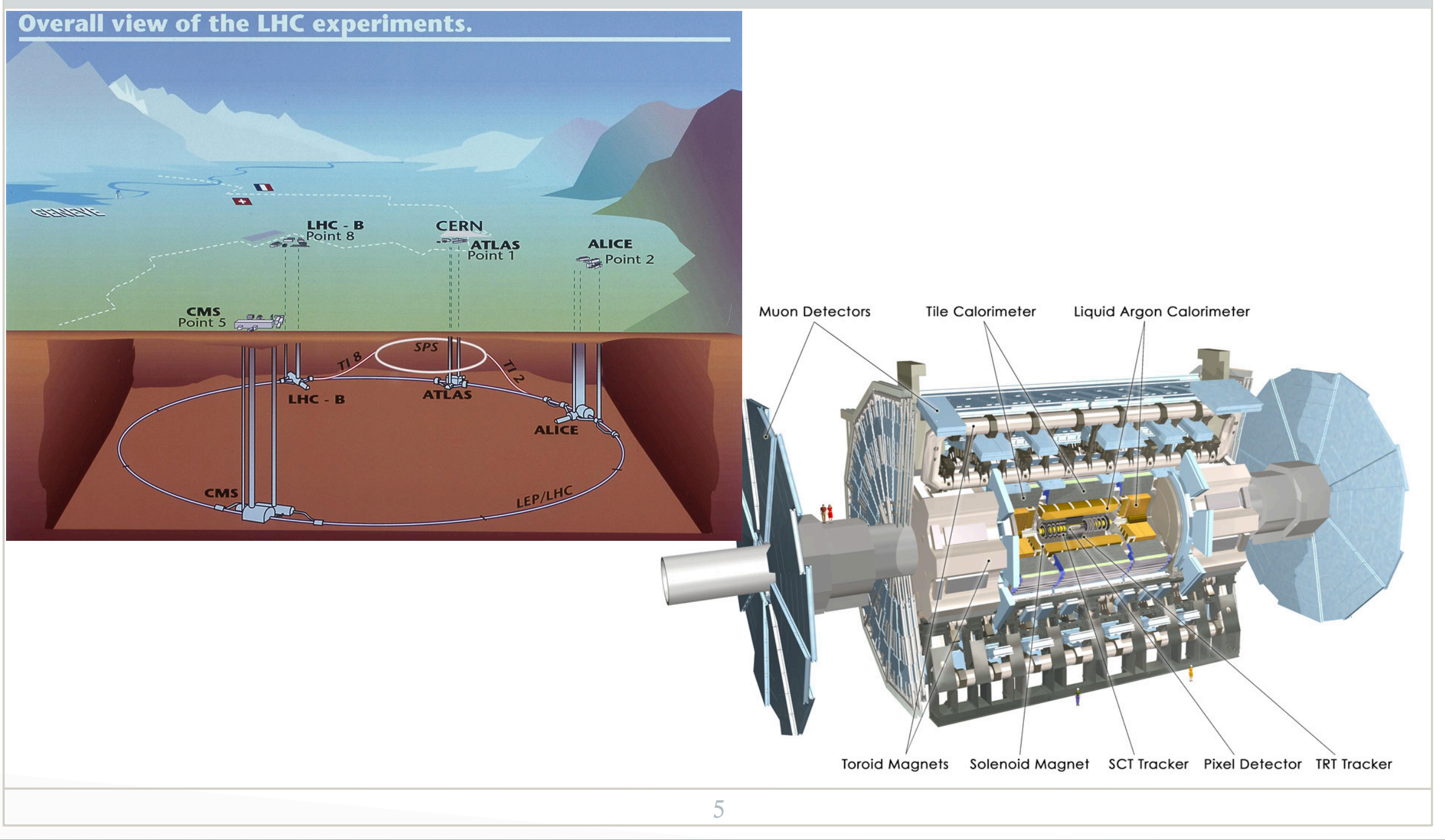




\section{Jets}

- What are jets?

- Jets are a cone of hadrons which are a produced by a quark or gluon hadronizing multiple times before it reaches the detector

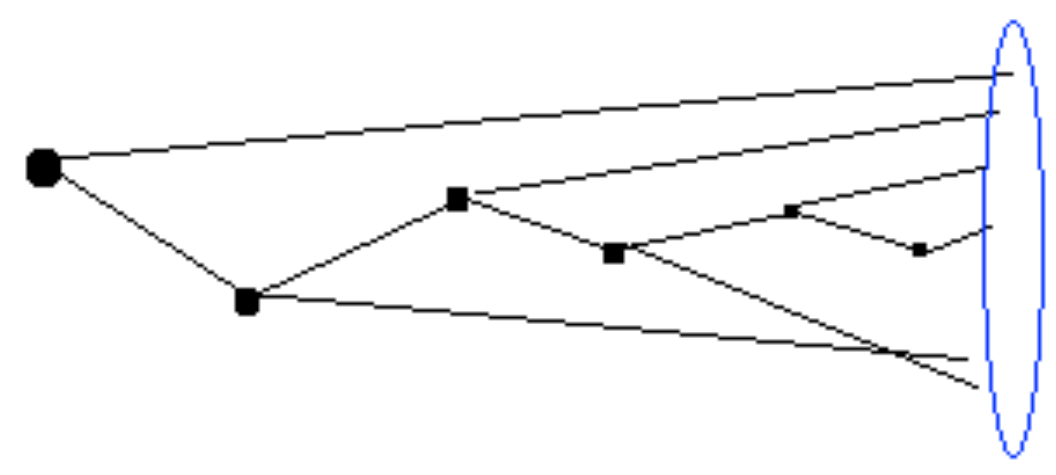




\section{Jets}

- What are jets?

- Jets are a cone of hadrons which are a produced by a quark or gluon hadronizing multiple times before it reaches the detector

\section{hadronization}

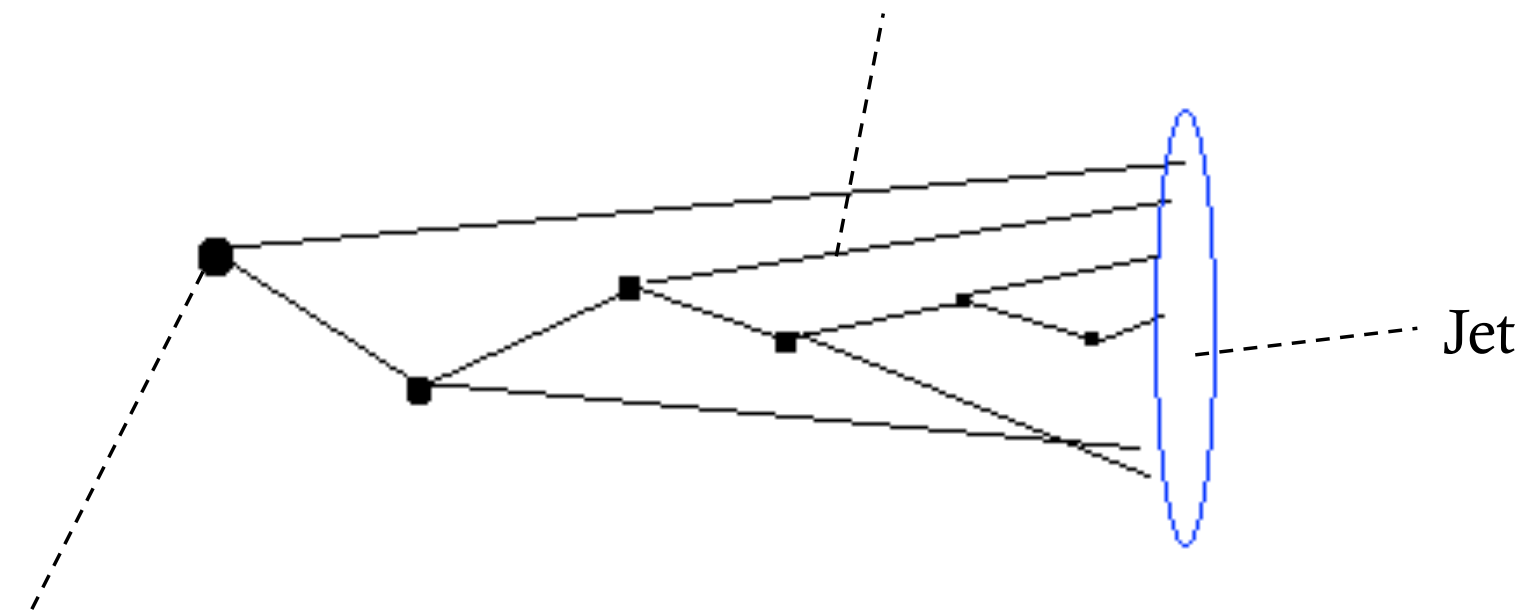

Incoming quark 


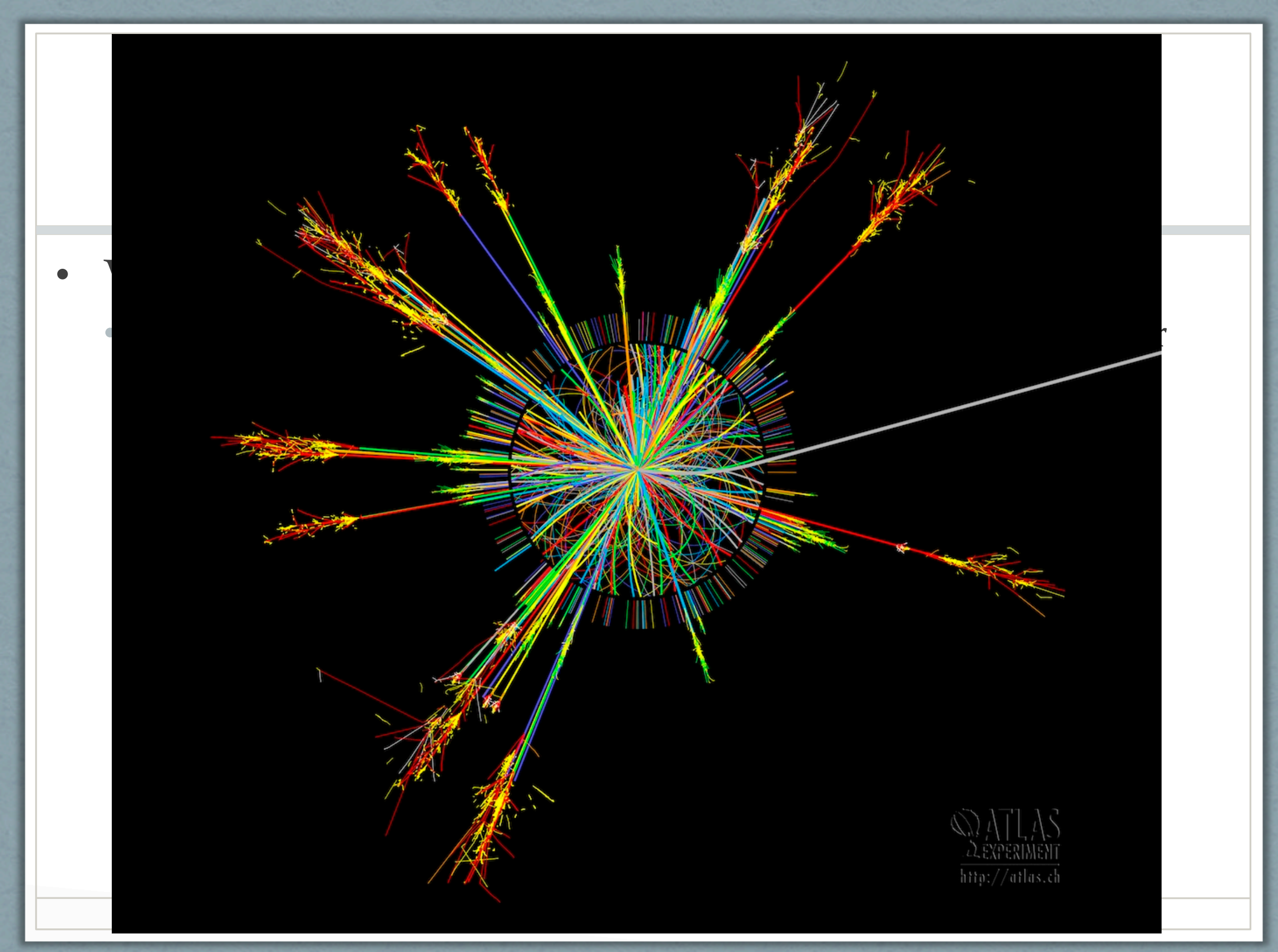




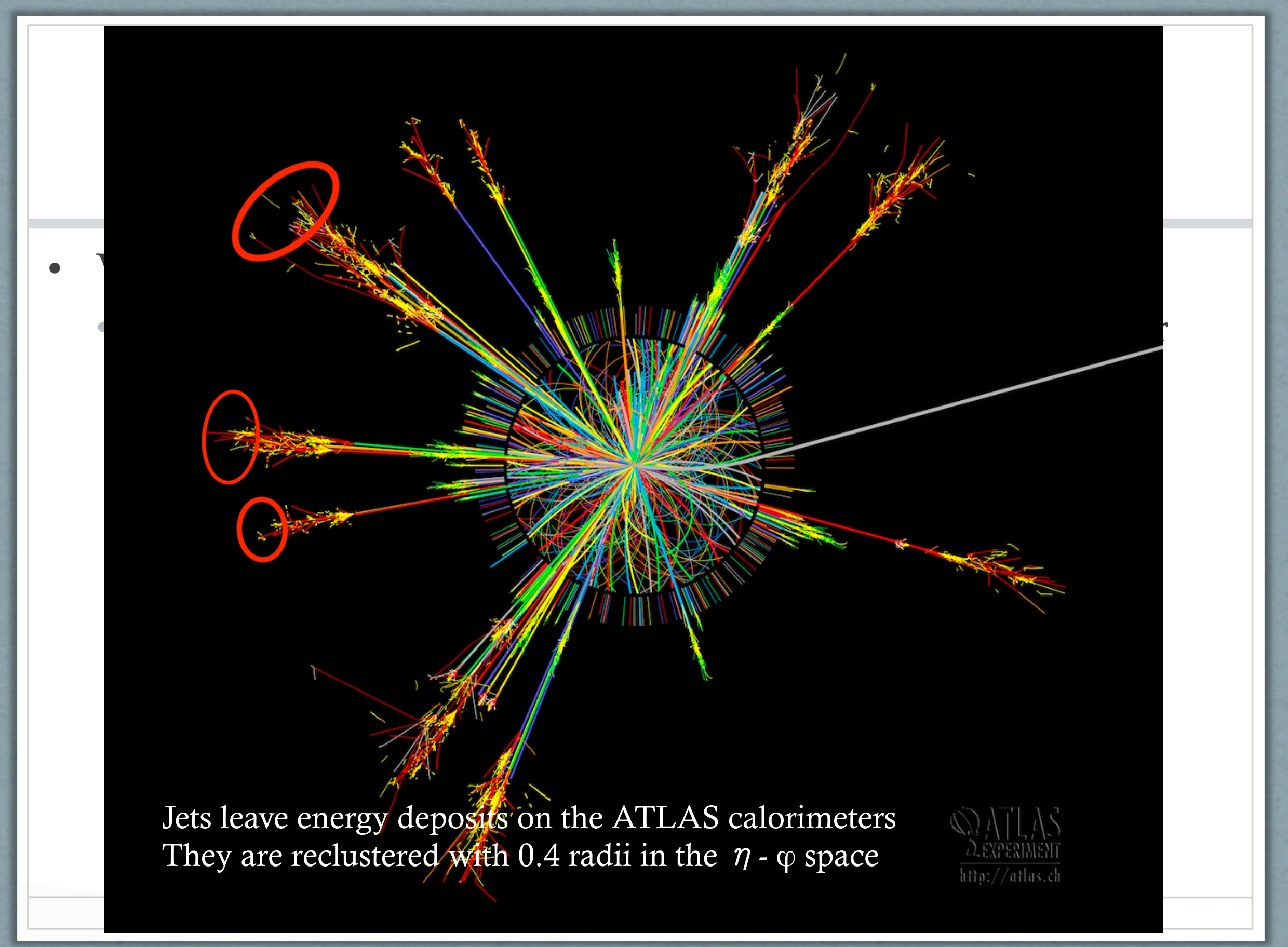




\section{Boosted Objects}

- When a top quark is highly boosted its decay products will be collimated

- The radius of the cone produced (jet) depends on the the $\mathrm{P}_{\mathrm{T}}$ of the top quark $\left(\mathrm{R}=2 \mathrm{M} / \mathrm{P}_{\mathrm{T}}\right)$

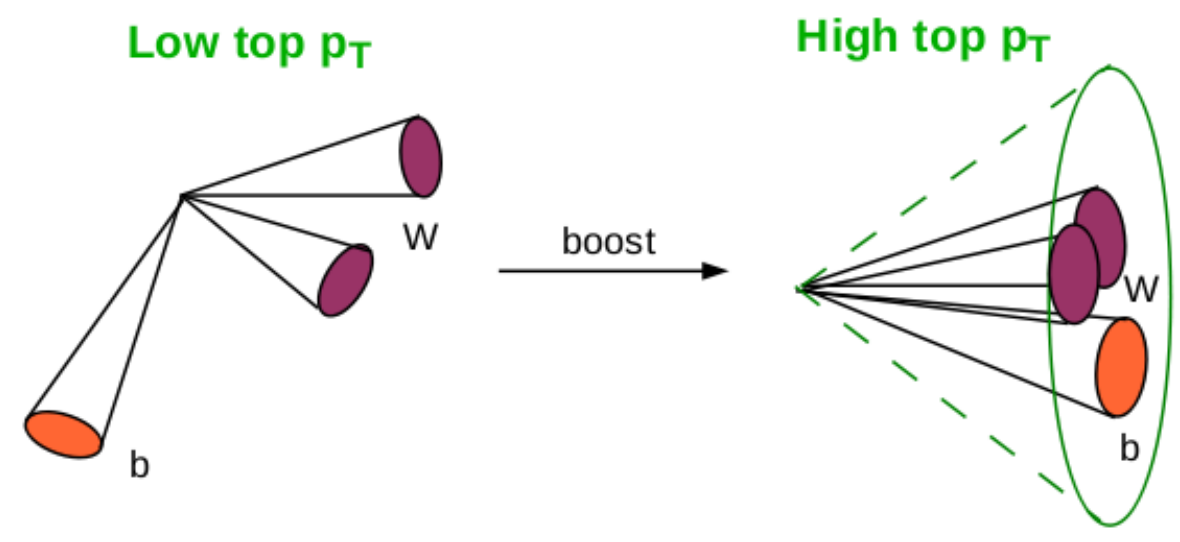




\section{Reclustering}

Jet reclustering allows jets to be calibrated at a small radius $r$, and then used as inputs for reconstructing larger jets of radius $R$

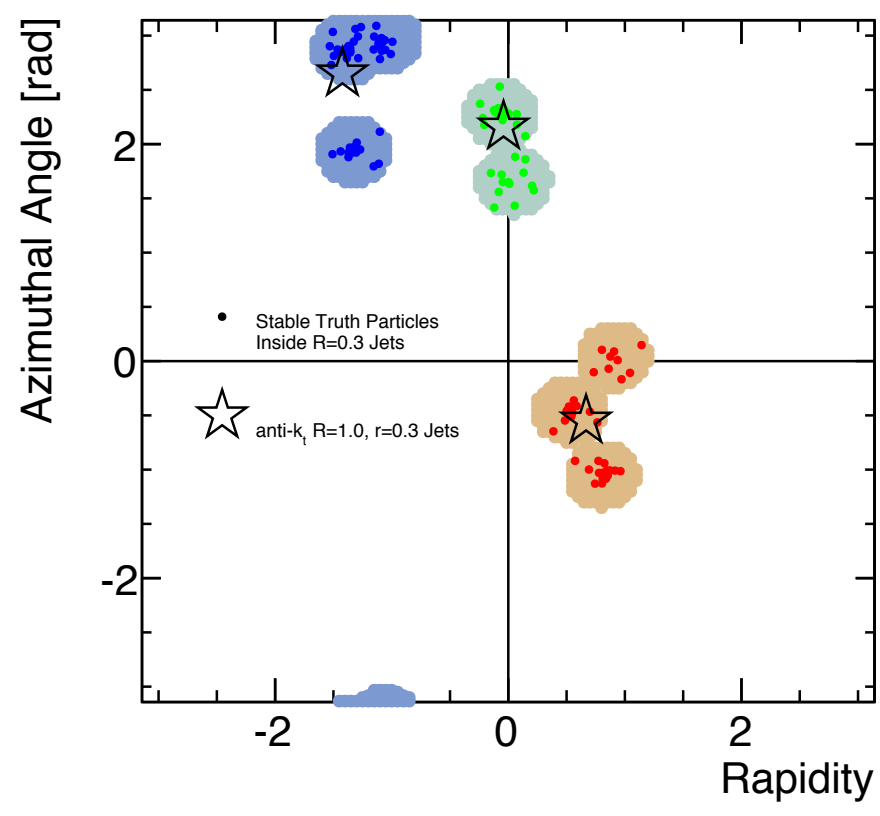

- Energy Deposit Jets

is Reclustered Jets

http://arxiv.org/pdf/1407.2922v2.pdf 


\section{VariableR vs Fixed}

Fixed Reclustering: uses jets of $r=0.4$ to reconstruct jets of $\mathrm{R}=1.0$

Variable Reclustering: uses a range from $\mathrm{R}_{\min }=0.4$ to $\mathrm{R}_{\max }=1.5$ to reconstruct jets

- VariableR Allows us to reconstruct jets of various kinematic regimes

- $\mathrm{R}=2 \mathrm{M} / \mathrm{PT}$ define typical scale for the jets which is given by:

- $\rho_{\mathrm{T}}=2 * 173.5 \quad \rho_{\mathrm{W}}=2 * 80.4$

http://arxiv.org/abs/0903.0392

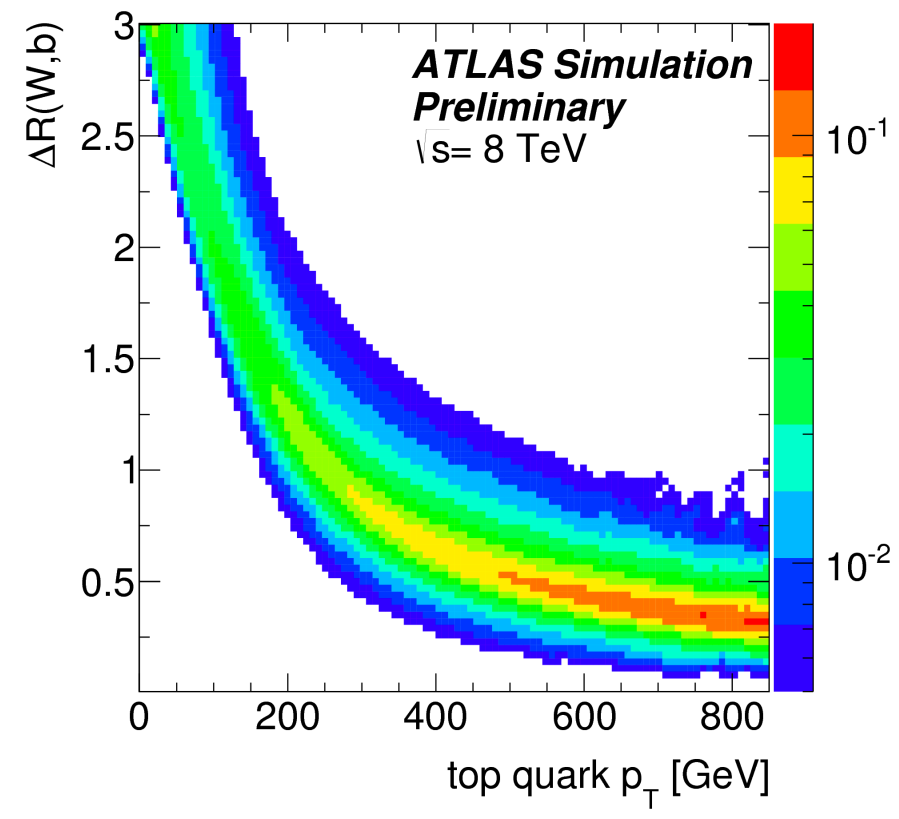




\section{Boosted Top Reconstruction}

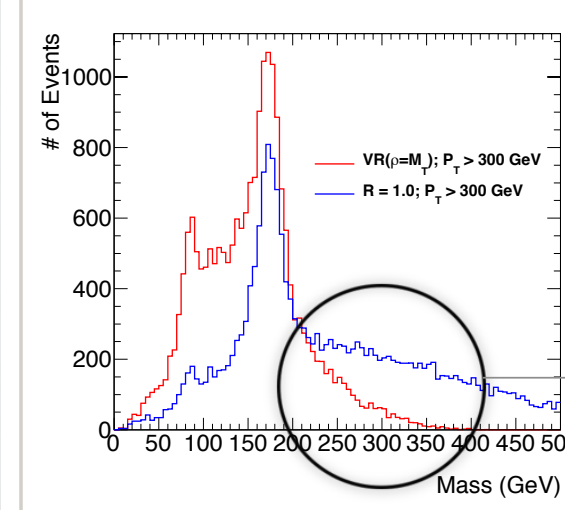

Mass distribution for

highest $\mathrm{P}_{\mathrm{T}}$ top quark

candidate

드

+ VR constituent

$\triangle$ Fixed constituent

O VR

O Fixed

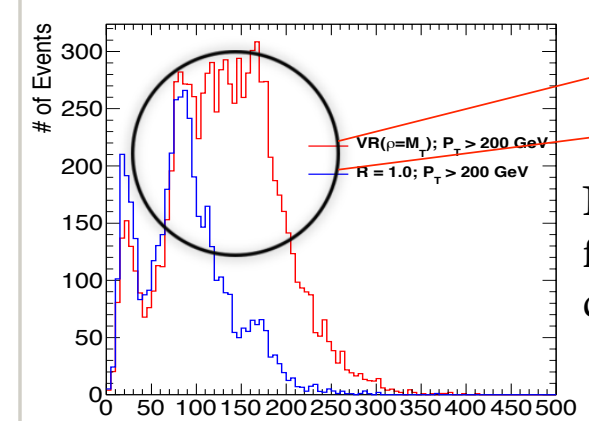

Mass (GeV)

Mass distribution for fourth highest $\mathrm{P}_{\mathrm{T}}$ top quark candidate
무은

$=$
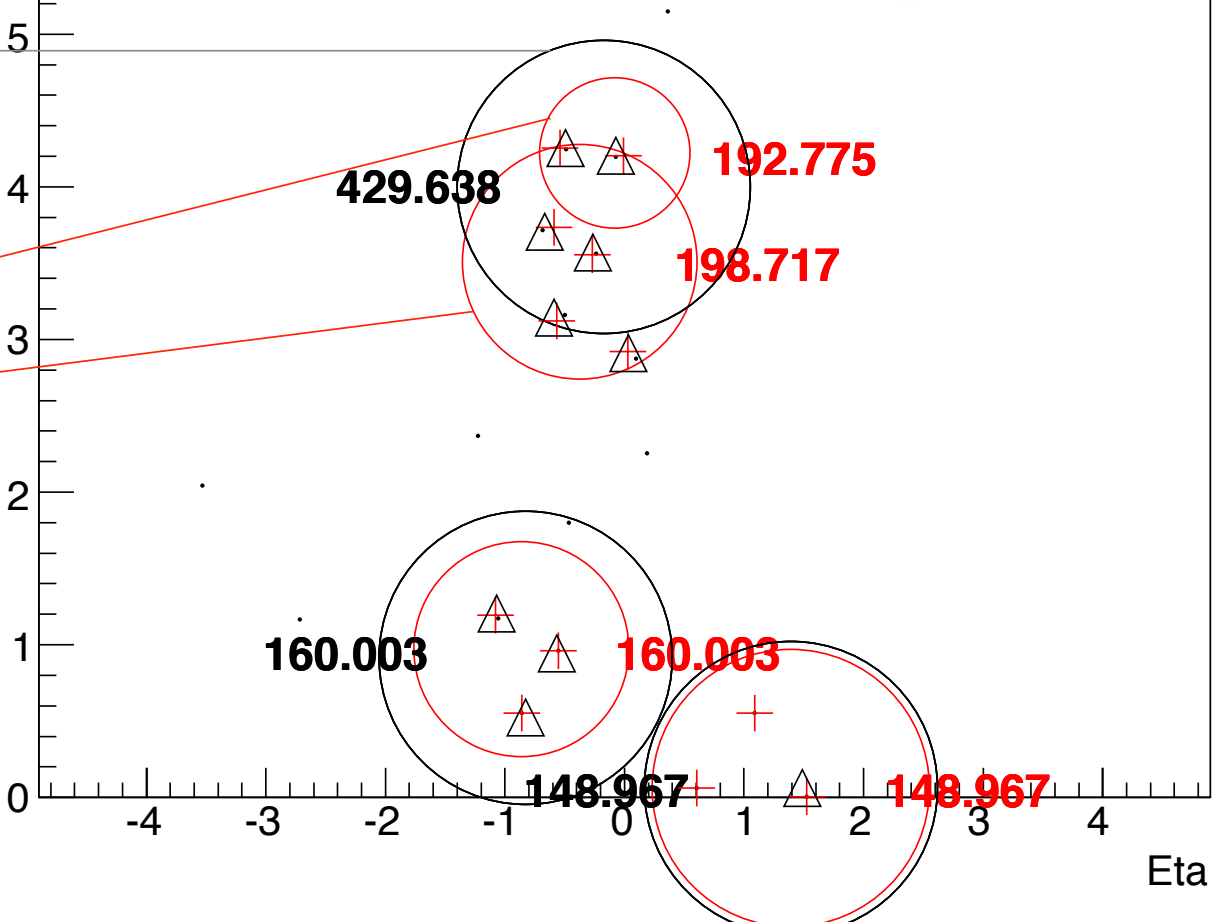


\section{Significance}
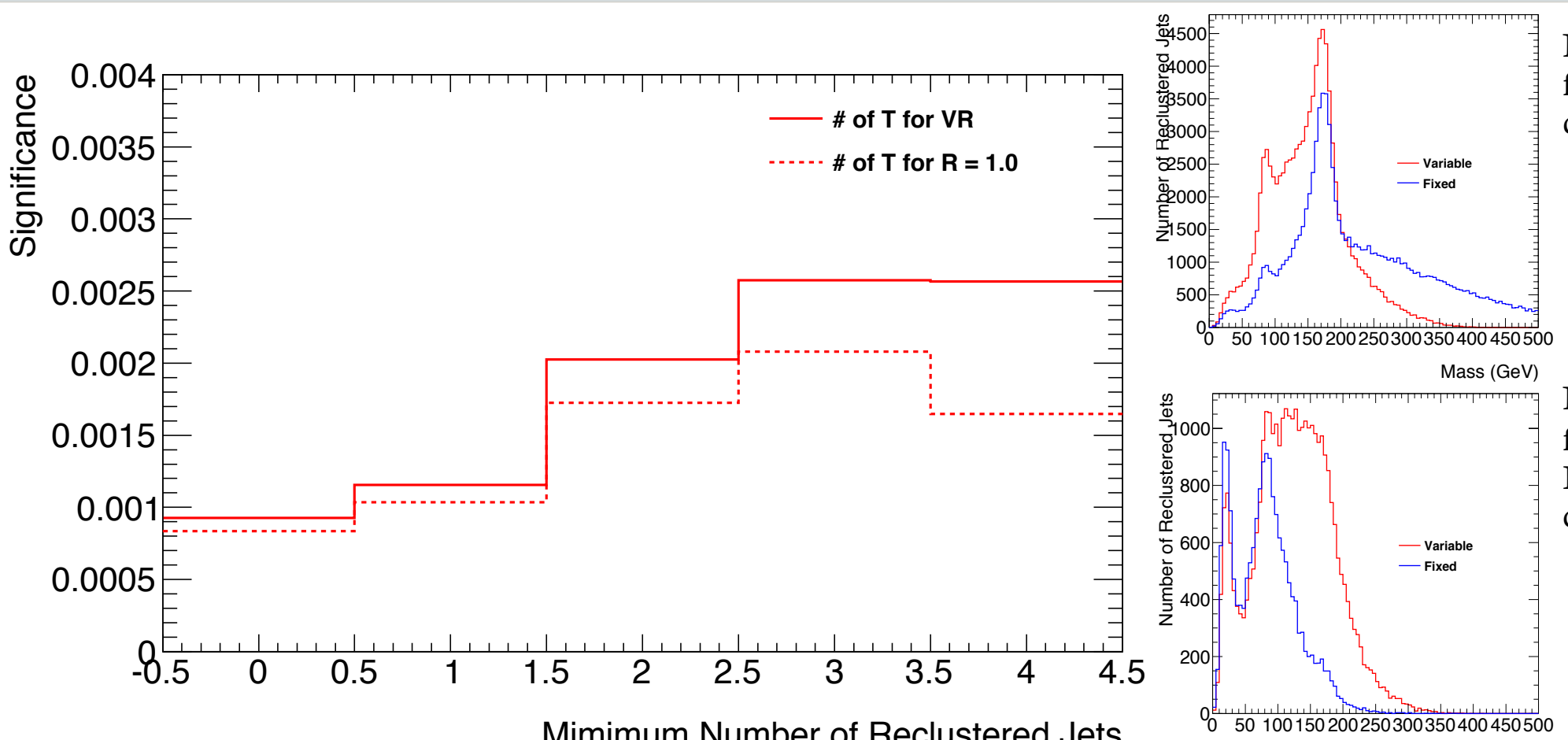

Mass distribution for highest $\mathrm{P}_{\mathrm{T}}$ top quark candidate

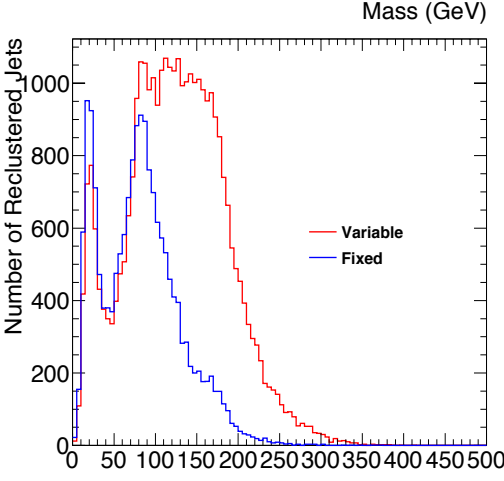

Mass distribution for fourth highest $\mathrm{P}_{\mathrm{T}}$ top quark candidate

Mass $(\mathrm{GeV})$

Significance $=\frac{\text { signal }}{\sqrt{\text { signal }+ \text { background }}}$

The VariableR algorithm is more efficient at reconstructing multiple jets and so allows us to discriminate against low top quark candidate multiplicity 


\section{Boosted W Reconstruction}

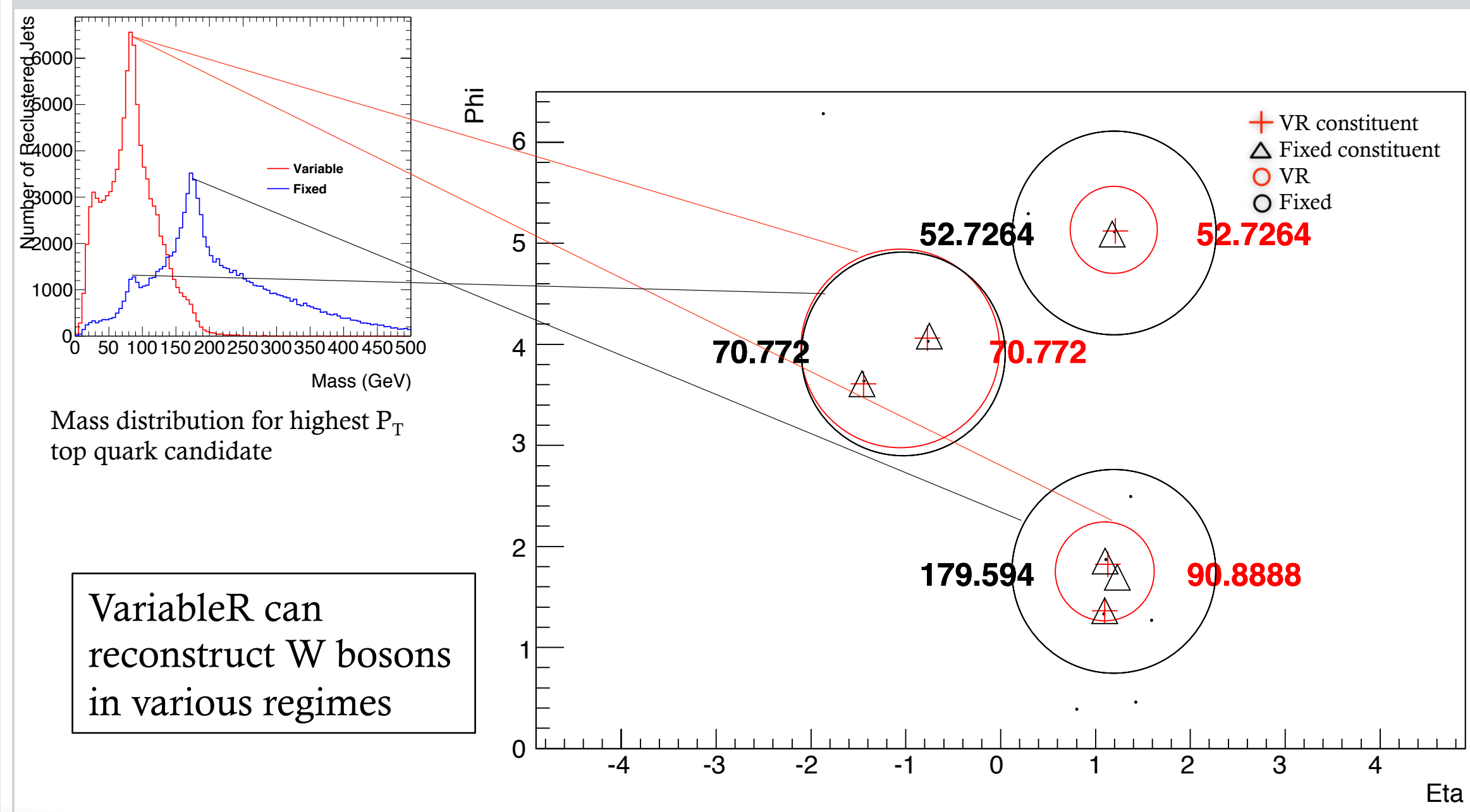




\section{Significance Comparison}

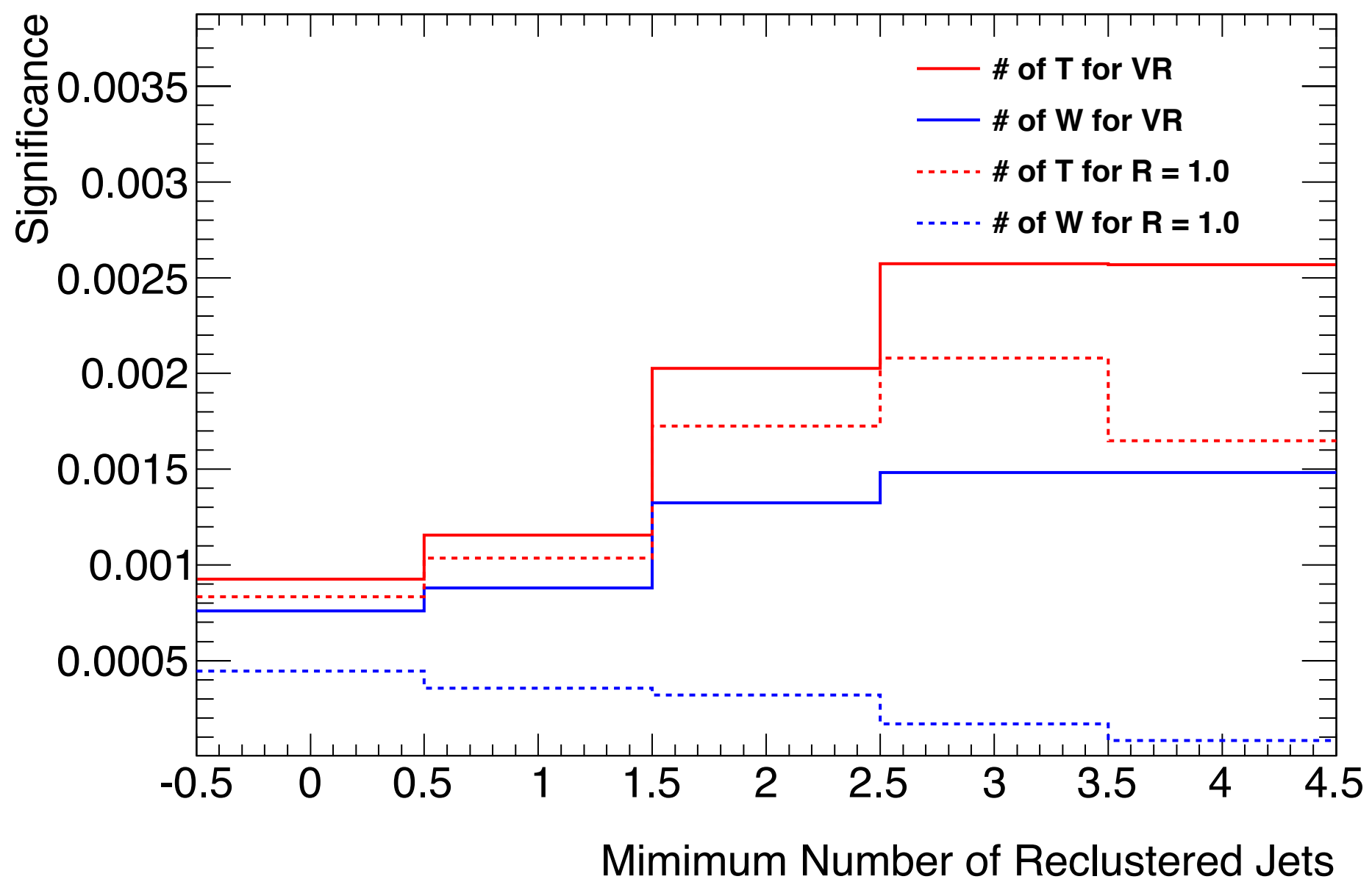




\section{Conclusions and Next Steps}

- I learned about reconstructing boosted top quarks and $\mathrm{W}$ bosons in the hadronic final state

- Studied the application of a new algorithm (VariableR reclustering) for identification of new physic processes

- This technique will be implemented in the super-symmetry search for gluino mediated stop pair production 


\section{Acknowledgements}

- Francesco Rubbo

- Ariel Schwartzman

- Ben Nachman

- Su Dong

- Fastjet.fr

- stackoverflow

- SULI, DOE, ATLAS and SLAC 